\title{
An algorithm had moderate sensitivity for identifying older women in nursing homes at risk of fracture
}

\author{
Girman CJ, Chandler JM, Zimmerman SI, et al. Prediction of fracture in nursing home residents.J Am Geriatr Soc \\ 2002;50:1341-7.

\section{QUESTION: Does an algorithm composed of routinely collected baseline data identify older women in nursing homes at increased risk of fracture?}

Sources of funding: Merck Research

Laboratories and

National Institute on

Aging.

For correspondence:

Dr C J Girman

Department of

Epidemiology, Merck

Research Laboratories,

Blue Bell, PA, USA.

girman@merck.com

\section{Design}

18 month follow up of a cohort of nursing home residents divided into derivation and validation samples.

\section{Setting}

A stratified random sample of 47 long term nursing facilities in Maryland, USA.

\section{Patients}

1427 white women living in nursing homes who were $\geq 65$ years of age (mean age $85 \mathrm{y}$ ), had no terminal cancer or bone metastases, were not comatose, had $\geq 1$ wrist

\section{COMMENTARY}

Injurious falls are a major problem in long term care facilities. The interaction of both intrinsic and extrinsic factors contributes to the risk of falls. The study by Girman et al focuses exclusively on intrinsic risk factors. A clinical algorithm to identify those at risk of fractures offers the possibility of allocating preventive efforts to those most in need. The study approach is intuitively appealing because it involves using routinely collected MDS data.

The algorithm did predict risk better than chance, but many women were still misclassified in terms of their level of risk. Several study limitations may have introduced "noise", limiting the precision of the proposed algorithm. Patients admitted to long term care may experience rapid functional decline. ${ }^{1}$ Thus, risk factors such as weight, transfer independence, and cognition may have shifted from the timing of the MDS assessment. Some important risk factors for fractures, such as the use of benzodiazepines ${ }^{2}$ and environmental characteristics, were excluded. Finally, the circumstances culminating in a fracture were not disclosed, but it is reasonable to assume that they often involved an interaction of MDS characteristics with environmental factors.

In an earlier report of this study, ${ }^{3}$ the risk of fracture was found to be higher among women with low BMD and transfer independence but not among those with low BMD and transfer dependence. This raises the question of which preventive measures should be routinely used for patients who are able to transfer independently in order to safely support their mobility independence and physical and social functioning. Characteristics of the physical environment (eg, night lighting, transfer boards, distance to bathroom for patients with urge incontinence, and use of physical restraints) and workload environment (eg, ratio of staff to patients and availability of staff) are critical areas for intervention.

It would be premature to adopt the use of the algorithm for the assessment of women at high risk of fracture in nursing homes. Further research is required to unravel the means by which transfer independence affects the risk of falls and consequently risk of fractures.

\section{Nancy Edwards, RN, PhD Professor and CHSRF/CIHR Nursing Chair School of Nursing, University of Ottawa Faranak Aminzadeh, RN, MScN Research Associate/Geriatric Assessor Regional Geriatric Assessment of Program of Ottawa Ottawa, Ontario, Canada}

1 Rubenstein LZ, Josephson KR, Osterweil D. Falls and fall prevention in the nursing home. Clin Geriatr Med 1996;12:881-902.

2 Ray WA, Thapa PB, Gideon P. Benzodiazepines and the risk of falls in nursing home residents. JAm Geriatr Soc 2000;48:682-5.

Chandler JM, Zimmerman SI, Girman CI, et al. Low bone mineral density and risk of fracture in white female nursing home residents. JAMA 2000;284:972-7. or forearm free of prosthetic implants and open skin lesions, were not admitted for rehabilitation only, and were able to have bone mineral density (BMD) measurements.

\section{Description of prediction guide}

The women's most recent minimum data set (MDS) (collected on all nursing home residents in the US) was the primary source of information for the algorithm (total 75 variables). The data pertained to physical functioning and activities of daily living, ambulatory status, vision status, mood and behaviour patterns, incontinence, dementia, other health conditions, and use of restraints. Classification and regression tree (CART) methodology and logistic regression were used to identify variables that would predict fractures. An additive scoring algorithm was derived in a random $67 \%$ of the cohort using cut points suggested by the CART methodology and validated in the remainder of the cohort.

\section{Main outcome measures}

Sensitivity and specificity of the algorithm for predicting osteoporotic fractures ascertained by physician note or $\mathrm{x}$ ray.

\section{Main results}

180 women $(12.6 \%)$ had osteoporotic fractures during follow up. Variables that contributed to the algorithm were body size (weight and height), mobility and transfer status, and activities of daily living. Age $\geq 75$ years, weight $<170$ pounds $(77 \mathrm{~kg})$, height $\leq 63$ inches $(160$ $\mathrm{cm}$ ), independent or supervised locomotion, fall in the past 180 days, dependence in $\leq 4$ selected activities of daily living, MDS cognition score $\leq 3$, and occasional incontinence contributed $\geq 1$ point to the score. The score was calculated by summing of the points. Using a cut point of $\geq 4$, the algorithm had moderate sensitivity but low specificity for predicting fracture in both the derivation and validation samples. In the derivation sample, the sensitivity was $79.7 \%$ and the specificity was $38.4 \%$; in the validation sample, the sensitivity was $70.2 \%$ and the specificity was $38.6 \%$. Fracture rates increased with increasing algorithm score and the c statistic (area under the receiver operating characteristic curve) was 0.63 .

\section{Conclusion}

An algorithm composed of routinely collected minimum data set baseline data had moderate sensitivity, but low specificity in predicting fractures in older white women in nursing homes. 